\title{
REGISTRATION OF PLANTAR PRESSURE IMAGES
}

\author{
Francisco P. M. Oliveira, João Manuel R. S. Tavares \\ Faculdade de Engenharia da Universidade do Porto (FEUP) / Instituto de Engenharia \\ Mecânica e Gestão Industrial (INEGI), Rua Dr. Roberto Frias, 4200-465 Porto, Portugal, \\ \{francisco.oliveira, tavares\}@fe.up.pt
}

\begin{abstract}
SUMMARY
We present an analysis of four different algorithms used to register plantar pressure images: a first one based on the matching of the external contours of the feet, a second algorithm based on the technique of phase correlation, a third one based on the direct optimization of the cross-correlation (CC) and using the Fourier transform, and a fourth and last algorithm that is based on the iterative optimization of an intensity (dis)similarity measure. In terms of accuracy, the later algorithm achieved the best registration results; on the other hand, the algorithm based on the matching of contours was the fastest, but its accuracy was inferior to the accuracy of the remaining algorithms.
\end{abstract}

Key Words: Pedobarography, Matching, Optimization, Cross-correlation, Fourier transform.

\section{INTRODUCTION}

Plantar pressure distribution provides significant information for clinicians and researchers about the structure and function of the foot and general mechanics of gait. It is, therefore, extremely helpful in the diagnosis of foot complaints, development of footwear [1] and to obtain useful information for gait analysis, just to mention some few examples. In fact, plantar pressure distribution allows the comparison of the loads in the limb, either between injured and non-injured or pre- and post-traumatic or -operative states, enables the comparisons between patients and control groups and provides detailed and specific information on each region of contact [2]. There are a number of different techniques to access the relevant pressure distribution, and, in the major part of them, the pedobarographic data can be converted into a discrete rectangular array. Therefore, techniques of image processing and analysis can be directly used on the converted data to aid both clinicians and researchers in order to obtain pertinent clinical information.

Image registration, the process of optimally aligning homologous structures represented in images, is demanded by clinicians and researchers, as some usual clinical tasks such as, cases comparison, identification of the main plantar pressure areas and classification of the foot type, can be easier attained. In addition, pedobarographic image registration supports pixel-level statistics, which makes possible the extraction of biomechanically-relevant information more effectively than traditional regional techniques [3].

Several studies on the registration of pedobarographic images have been developed; for example, using principal axes transformation [4], modal matching [5, 6], principal axes combined with steepest descent gradient search [7], optimization with evolutionary algorithms [8], based on foot size and progression angle [9], contours matching [10], optimization of the cross-correlation (CC) computed in the frequency domain [11], phase correlation [11], and optimization of an image 
(dis)similarity measure using an iterative scheme [12]. In this work, the later four methodologies are studied; thus, their fundamentals are introduced and a discussion about their results is presented.

\section{METHODS}

\subsection{Registration based on the matching of contours' points}

The registration algorithm presented in [10] is based on the matching of the external contours of the feet represented in the input images, and can be described in the following steps (Figure 1): 1) extraction of the external contours; 2) computation of an affinity matrix between the contours' points; 3) searching for the best matching by minimizing the sum of the affinities; 4) computation of the geometric transformation that best register the matched points; 5) registration of the input images considering the transformation computed.

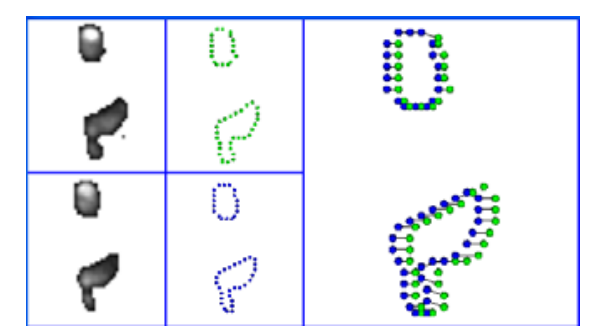

Figure 1: Matching the contours of two input images: on the left, two images to be registered; on the middle, extracted contours after a sampling process; and on the right, the matching found.

\subsection{Registration based on the technique of phase correlation}

The technique of phase correlation is based on the shift property of the Fourier transform; that is, a shift of a function in the space domain is represented by a shift on its phase when the function is represented in the frequency domain.

Let us consider two images, $f$ and $g$, and their Fourier transforms, $F$ and $G$, respectively, and suppose that $g(x, y)=f\left(x-x_{0}, y-y_{0}\right)$; thus, according to the shift property [13], we can get:

$$
G(u, v)=F(u, v) e^{-2 \pi i\left(u x_{0}+v y_{0}\right)} .
$$

Then, by computing the cross-power, we have:

$$
\frac{F(u, v) G^{*}(u, v)}{\left|F(u, v) G^{*}(u, v)\right|}=e^{-2 \pi i\left(u x_{0}+v y_{0}\right)},
$$

where $G^{*}$ represents the complex conjugate of $G$.

By computing the inverse of the Fourier transform of the cross-power, a Dirac $\delta$-distribution centered at $\left(x_{0}, y_{0}\right)$ is achieved. Therefore, the coordinates of the Dirac pulse indicate the optimal integer shift.

The aforementioned procedure allows the determination of the shift, but requires that the scale and rotation are known. To obtain the required values, first the spectrums of both images are built and converted into the log-polar coordinate system. Then, by determining the shift between the logpolar spectrums using the phase correlation, the scale and rotation can be known [11].

\subsection{Registration based on the optimization of an image intensity (dis)similarity measure}

This family of registration methodologies is based on the optimization of an image (dis)similarity measure related to the pixels' intensities. Thus, the geometric transformation that optimizes the (dis)similarity measure considered is then used to register the input images. 
A two-step approach is considered in [12] to optimize the (dis)similarity measure adopted: in the begin, is determined a pre-registration; afterwards, in the second step, the optimization algorithm starts from the pre-registration solution searching iteratively for the geometric transformation that optimizes the adopted (dis)similarity measure. In that framework, for the pre-registration the user can choose the matching of the represented contours [10], the correlation of the images' phases or the cross-correlation of the input images [11]. Additionally, the Powell's method is adopted in the optimization process of the second step, taking into account one of the following (dis)similarity measures: the mean squared error (MSE), the mutual information (MI) or one based on the exclusive-or (XOR) [8].

In [12] the (dis)similarity measures are optimized using an iterative optimization algorithm. However, as shown in [11], for the CC and sum of the squared differences (SSD) (dis)similarity measures, the optimal integer shift, scaling and rotation values can be obtained directly by using the Fourier transform and convolution theorem. Let us consider two images, $f$ and $g$, and their CC in function of a shift, $a$ :

$$
\mathrm{CC}_{f g}(a)=\sum_{i} f(i) g(i-a) .
$$

By the convolution definition, one have:

$$
\mathrm{CC}_{f g}(a)=\sum_{i} f(i) \bar{g}(a-i)=\{f * \bar{g}\}(a),
$$

where $\bar{g}(i)=g(-i)$ and $*$ represents the convolution. Then, from the convolution theorem, one can obtain:

$$
\mathbf{F}\{f * \bar{g}\}=k \cdot \mathbf{F}\{f\} \cdot \mathbf{F}\{\bar{g}\},
$$

where $\mathbf{F}$ represents the Fourier transform and $k$ is a constant that depends on the specific Fourier transform normalization.

Therefore, computing the inverse of the Fourier transform (using, for instance, the inverse fast Fourier transform (IFFT)) of the product in Equation (5), the correlation can be obtained for all shifts. Then, the coordinates of the point that has the higher value represents the desired integer optimal shift. On the other hand, to achieve the optimal scaling and rotation values, a procedure similar to the one refereed in section 2.2 can be used.

To directly obtain the similarity geometric transformation, that is, the scaling, rotation and shift values, which optimizes the SSD, a procedure similar to the one used to optimize the CC is employed [11].

\section{DISCUSSION}

All introduced algorithms were tested on a dataset consisting of 30 pairs of peak pressure images of $45 \times 63$ pixels, collected at $500 \mathrm{~Hz}$ using a $0.5 \mathrm{~m}$ Footscan system (RSscan, Olen, Belgium).

From the experimental results [10-12], we can point out the following conclusions. All algorithms revealed high accuracy, speed and noise robustness, being the most accurate the one presented in [12] that is based on a two-steps registration scheme and iterative optimization. This result was expected, since the second step of this algorithm starts from the pre-registration obtained by one of the remains algorithms.

In respect to the image (dis)similarity measure used in the algorithm presented in [12], when a known control geometric transformations were used to assess the registration accuracy, the best results were obtained by minimizing the MSE, being the associated residual error (RE) always inferior to $10^{-4} \mathrm{~mm}$. However, very good results were also obtained when the MI was minimized $(\mathrm{RE}<0.015 \mathrm{~mm})$, and suitable results were obtained when the CC was directly maximized (0.041 $\mathrm{mm}$ ) [12]. Considering the same known control geometric transformations, but after adding Gaussian noise, with a signal to noise ratio (SNR) between 1.5 and 3.5, to the images to be register, the maximum RE remained very low $(<0.4 \mathrm{~mm})$ [12]. 
Finally, the processing time was always very low for the four algorithms, which were implemented in $\mathrm{C}++$ and tested on a notebook PC with an AMD Turion64 2.0 GHz microprocessor and 1.0GB of RAM. The fastest algorithm was the one based on the matching of the represented contours (around 25 ms per image pair), and the slowest was the algorithm based on the two-steps registration scheme (around $70 \mathrm{~ms}$ ) [12].

\section{Acknowledgements}

This work was partially done in the scope of the projects "Methodologies to Analyze Organs from Complex Medical Images - Applications to Female Pelvic Cavity", "Aberrant Crypt Foci and Human Colorectal Polyps: mathematical modelling and endoscopic image processing” and "Cardiovascular Imaging Modeling and Simulation - SIMCARD", with references, PTDC/EEACRO/103320/2008, UTAustin/MAT/0009/2008 and UTAustin/CA/0047/2008, respectively, financially supported by Fundação para a Ciência e a Tecnologia in Portugal.

The first author would like to thank Fundação Gulbenkian, in Portugal, for his PhD grant.

\section{REFERENCES}

[1] R. L. Actis, et al., "Multi-plug insole design to reduce peak plantar pressure on the diabetic foot during walking," Medical \& Biological Engineering \& Computing, 46:363-371, 2008.

[2] D. Rosenbaum and H. Becker, "Plantar pressure distribution measurements. Technical background and clinical applications," Foot and Ankle Surgery, 3:1-14, 1997.

[3] T. C. Pataky, et al., "New insights into the plantar pressure correlates of walking speed using pedobarographic statistical parametric mapping," Journal of Biomechanics, 41:1987-1994, 2008.

[4] A. J. Harrison and P. J. Hillard, "A moment-based technique for the automatic spatial alignment of plantar pressure data," Proceedings of the Institution of Mechanical Engineers, Part H: Journal of Engineering in Medicine, 214:257-264, 2000.

[5] L. F. Bastos and J. M. R. S. Tavares, "Improvement of modal matching image objects in dynamic pedobarography using optimization techniques," Articulated Motion And Deformable Objects: Third International Workshop, Amdo 2004, Spain, 39-50, 2004.

[6] R. R. Pinho and J. M. R. S. Tavares, "Dynamic pedobarography transitional objects by Lagrange's equation with FEM, modal matching and optimization techniques," Image Analysis and Recognition: International Conference, ICIAR 2004, Portugal, 92-99, 2004.

[7] T. C. Pataky and J. Y. Goulermas, "Pedobarographic statistical parametric mapping (pSPM): a pixel-level approach to foot pressure image analysis," Journal of Biomechanics, 41:2136-2143, 2008.

[8] T. C. Pataky, et al., "A comparison of seven methods of within-subjects rigid-body pedobarographic image registration," Journal of Biomechanics, 41:3085-3089, 2008.

[9] N. L. W. Keijsers, et al., "A new method to normalize plantar pressure measurements for foot size and foot progression angle," Journal of Biomechanics, 42:87-90, 2009.

[10] F. P. M. Oliveira, et al., "Rapid pedobarographic image registration based on contour curvature and optimization," Journal of Biomechanics, 42:2620-2623, 2009.

[11] F. P. M. Oliveira, et al., "Registration of pedobarographic image data in frequency domain," Computer Methods in Biomechanics and Biomedical Engineering, (in press).

[12] F. P. M. Oliveira and J. M. R. S. Tavares, "Novel framework for registration of pedobarographic image data," Medical \& Biological Engineering \& Computing, (in press).

[13] E. Castro and C. Morandi, "Registration of translated and rotated images using finite Fourier transforms," IEEE Transactions on Pattern Analysis and Machine Intelligence, 9:700-703, 1987. 